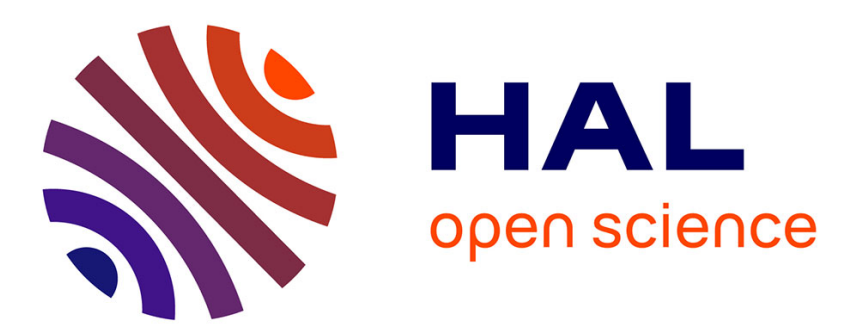

\title{
Revisiting Recency Abstraction for JavaScript Towards an Intuitive, Compositional, and Efficient Heap Abstraction
}

Jihyeok Park, Xavier Rival, Sukyoung Ryu

\section{- To cite this version:}

Jihyeok Park, Xavier Rival, Sukyoung Ryu. Revisiting Recency Abstraction for JavaScript Towards an Intuitive, Compositional, and Efficient Heap Abstraction. SOAP 2017 - International Workshop on the State Of the Art in Java Program Analysis, Jun 2017, Barcelona, Spain. pp.1-6, 10.1145/3088515.3088516 . hal-01648682

\section{HAL Id: hal-01648682 \\ https://hal.inria.fr/hal-01648682}

Submitted on 27 Nov 2017

HAL is a multi-disciplinary open access archive for the deposit and dissemination of scientific research documents, whether they are published or not. The documents may come from teaching and research institutions in France or abroad, or from public or private research centers.
L'archive ouverte pluridisciplinaire HAL, est destinée au dépôt et à la diffusion de documents scientifiques de niveau recherche, publiés ou non, émanant des établissements d'enseignement et de recherche français ou étrangers, des laboratoires publics ou privés. 


\section{Revisiting Recency Abstraction for JavaScript}

\section{Towards an Intuitive, Compositional, and Efficient Heap Abstraction}

\author{
Jihyeok Park \\ KAIST, Republic of Korea \\ jhpark0223@kaist.ac.kr
}

\author{
Xavier Rival \\ DIENS, École Normale Supérieure, CNRS, \\ PSL Research University and INRIA, France \\ Xavier.Rival@ens.fr
}

\author{
Sukyoung Ryu \\ KAIST, Republic of Korea \\ sryu.cs@kaist.ac.kr
}

\begin{abstract}
JavaScript is one of the most widely used programming languages. To understand the behaviors of JavaScript programs and to detect possible errors in them, researchers have developed several static analyzers based on the abstract interpretation framework. However, JavaScript provides various language features that are difficult to analyze statically and precisely such as dynamic addition and removal of object properties, first-class property names, and higher-order functions. To alleviate the problem, JavaScript static analyzers often use recency abstraction, which refines address abstraction by distinguishing recent objects from summaries of old objects. We observed that while recency abstraction enables more precise analysis results by allowing strong updates on recent objects, it is not monotone in the sense that it does not preserve the precision relationship between the underlying address abstraction techniques: for an address abstraction $A$ and a more precise abstraction $B$, recency abstraction on $B$ may not be more precise than recency abstraction on $A$. Such an unintuitive semantics of recency abstraction makes its composition with various analysis sensitivity techniques also unintuitive. In this paper, we propose a new singleton abstraction technique, which distinguishes singleton objects to allow strong updates on them without changing a given address abstraction. We formally define recency and singleton abstractions, and explain the unintuitive behaviors of recency abstraction. Our preliminary experiments show promising results for singleton abstraction.
\end{abstract}

CCS Concepts - Software and its engineering $\rightarrow$ General programming languages; • Theory of computation $\rightarrow$ Program analysis

Permission to make digital or hard copies of all or part of this work for personal or classroom use is granted without fee provided that copies are not made or distributed for profit or commercial advantage and that copies bear this notice and the full citation on the first page. Copyrights for components of this work owned by others than ACM must be honored. Abstracting with credit is permitted. To copy otherwise, or republish, to post on servers or to redistribute to lists, requires prior specific permission and/or a fee. Request permissions from Permissions@acm.org.

SOAP'17, June 18, 2017, Barcelona, Spain

(C) 2017 ACM. 978-1-4503-5072-3/17/06 $\$ 15.00$

http://dx.doi.org/10.1145/3088515.3088516
Keywords Address abstraction, recency abstraction, address partition

\section{Introduction}

JavaScript is one of the most widely used programming languages. It is now the 7th popular language [1] and it becomes the de facto language for web programming. In the evergrowing IoT era, the realm of JavaScript may expand even more [2] and understanding and detecting bugs in JavaScript programs are getting more important.

Recently, researchers have presented several static analyzers for JavaScript programs. SAFE [7], TAJS [5], and WALA [10] statically analyze JavaScript programs based on the abstract interpretation framework. Because they aim for sound static analysis, their analysis results are often imprecise. Thus, each analyzer develops its own analysis techniques to improve the analysis precision $[3,8,10,11]$.

For JavaScript static analysis, analyzing "object properties" precisely serves an important role in improving the analysis precision. First, because property names themselves are first-class values, imprecise analysis of property names lead to imprecise analysis of property accesses. Second, since object properties may be added or removed dynamically, precisely analyzing the existence of object properties is challenging. Imprecisely analyzing that a specific property may not exist in an object may result in reporting a false type error. Third, because JavaScript supports higher-order functions, the values of object properties may be functions, which implies that building control flow graphs precisely requires precise analysis of property accesses.

To analyze object properties more precisely, JavaScript static analyzers often use recency abstraction [4]. Note that one of main causes of the analysis imprecision is weak update, which updates the value of an object property to a join of its old value and a new value. To analyze such updates more precisely, recency abstraction distinguishes the most recently allocated objects from joined old objects and performs weak updates on joined old objects and strong updates that replace old values with new values on the recently allocated objects. Thus, recency abstraction enhances the analysis precision for the most recently allocated objects. 
Recency abstraction is yet another address abstraction that divides a given (underlying) partition-based address abstraction into two parts: old and recent addresses. A partition-based address abstraction divides addresses into partitions and uses their powersets as its abstract domain. One example partition-based address abstraction is the allocation-site abstraction, which creates partitions by merging all objects created at the same allocation sites. For each partition, recency abstraction distinguishes a recent address that points to the most recently created objects and an old address that points to old objects, and it allows strong updates only on recent addresses. Consider the following code:

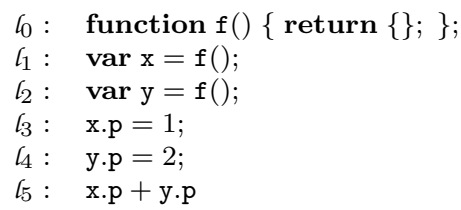

Though it is contrived for brevity, it shows how recency abstraction for the allocation-site abstraction works succinctly. Since the values of $\mathrm{x}$ and $\mathrm{y}$ are objects created at the same allocation site, $\iota_{0}$, both $\mathrm{x}$ and y have the same partition $l_{0}$ in the allocation-site abstraction. Thus, at the end of the above code, both x.p and y.p have undefined (the default value for an absent property), 1 , and 2 as their values. On the contrary, recency abstraction splits $\complement_{0}$ into two parts: $\left(\mathcal{L}_{0}, \mathbf{o}\right)$ for joined old addresses and $\left(\digamma_{0}, \mathbf{r}\right)$ for a recent address. At the end of the above code, $x$ has the old abstract address $\left(\mathcal{L}_{0}, \mathbf{o}\right)$ and $\mathrm{y}$ has the recent abstract address $\left(\mathcal{L}_{0}, \mathbf{r}\right)$. Thus, x.p has both undefined and 1 as its values because of weak updates on the old address, but y.p has only 2 as its value because of the strong update on the recent address.

While recency abstraction provides more precise analysis than its underlying address abstraction, it is not monotone in the sense that it does not preserve the refinement relationship between its underlying address abstraction techniques. We say that a partition-based address abstraction $A_{1}$ with a partition $\delta_{1}$ is a refinement of another partition-based address abstraction $A_{2}$ with a partition $\delta_{2}$, if the partition $\delta_{1}$ is finer than the partition $\delta_{2}$. We prove that the refinement relationship is proportional to the analysis precision. Unfortunately, recency abstraction on $A_{1}$, which is a refinement of $A_{2}$, may not be a refinement of recency abstraction on $A_{2}$. Thus, it is unclear which address abstraction would provide the most precise analysis in conjunction with recency abstraction, which denotes that recency abstraction is not compositional with other analysis techniques.

In this paper, we present a singleton abstraction, which improves the analysis precision of its underlying address abstraction without the aforementioned problems of recency abstraction. The contributions of this paper are as follows:

- We formally define recency abstraction on a partitionbased address abstraction such as the allocation-site abstraction, and describes how it interferes with address partitioning and analysis sensitivities.

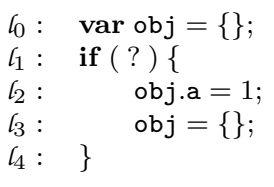

Figure 1. A simple example program

- We propose a singleton abstraction, which enhances the analysis precision while preserving the refinement relationship of its underlying address abstraction. Therefore, it is compositional with other analysis techniques.

- Our preliminary experimental results show that the singleton abstraction provides similar analysis precision as recency abstraction.

\section{Concrete Semantics}

In this section, we define the concrete semantics of a simplified variant of JavaScript. It contains essential constructs for address abstraction, and we augment the standard concrete semantics with time information to identify recently created objects. We call such time information a date, which is a non-negative integer.

\subsection{Notations and Syntax}

We use the following notations:

$\begin{array}{rllll}l & \in & \mathbb{L} & : & \text { control states } \\ \mathrm{x}, \mathrm{y} & \in & \mathbb{X} & : & \text { variables } \\ a & \in & \mathbb{A} & : & \text { addresses } \\ & & \mathbb{V}_{\mathrm{p}} & : & \text { primitive values } \\ & & \mathbb{V} & : & \text { values }\left(\mathbb{V}=\mathbb{A} \uplus \mathbb{V}_{\mathrm{p}}\right) \\ & & \mathbb{D} & : & \text { dates (non-negative integers) } \\ & \mathbb{P} & : & \text { property names of objects (strings) }\end{array}$

We let $\odot$ denote the undefined value $\left(\odot \in \mathbb{V}_{\mathrm{p}}\right)$. A date denotes when an object is created used for recency abstraction. Property names are string values. We consider the following abstract syntax as a simplified variant of JavaScript:

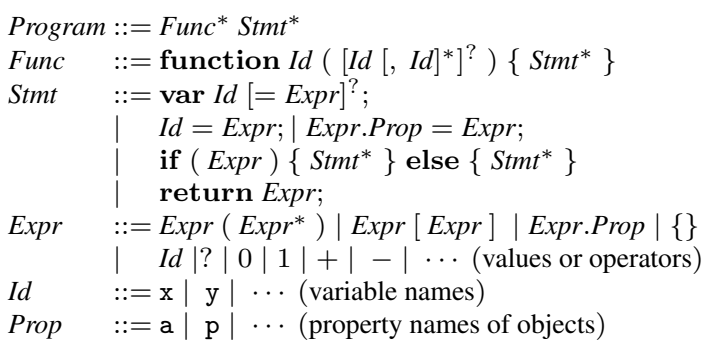

We use ? to denote unknown values such as dynamically generated values. Figure 1 shows an example code. Given a statement $\mathrm{s}$, we write $\zeta_{0}: \mathrm{s} ; \mathcal{l}_{1}$, if $\zeta_{0}$ is the control state right before the statement and $l_{1}$ is the control state right after it.

\subsection{States and Traces}

A state $\sigma=\left(\sigma^{\mathbb{L}}, \sigma^{\mathbb{C}}, \sigma^{\mathbb{H}}, \sigma^{\mathbb{D}}\right) \in \mathbb{S}$ consists of a control state, a context, a heap, and a date: 


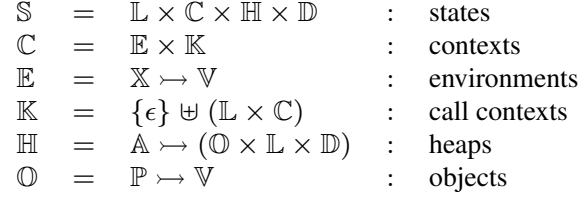

A context consists of an environment and a call context. An environment is a partial map from variables to values. A call context in top-level is $\epsilon$; in a function $\mathrm{f}$, a call context is a pair of the control point and the context of the call-site of $f$. A heap is a partial map from addresses to objects with their allocation sites and dates. An object is a partial map from property names to their values.

A trace $\tau \in \mathbb{T}$ is a finite sequence of states $\left\langle\sigma_{0}, \ldots, \sigma_{n-1}\right\rangle$. The date of a state captures the number of program execution steps so far: in a well-formed trace $\left\langle\sigma_{0}, \ldots, \sigma_{n-1}\right\rangle$, the date of $\sigma_{i}$ is $i$ and the transition rules in the concrete semantics defined in Section 2.3 ensure this. The following table represents sample traces for the example code in Figure 1. A trace executing the true branch is as follows:

\begin{tabular}{c|c|c|c}
$\sigma_{i}^{\mathbb{L}}$ & $\sigma_{i}^{\mathbb{E}}(\mathrm{obj})$ & $\sigma_{i}^{\mathbb{H}}$ & $\sigma_{i}^{\mathbb{D}}$ \\
\hline$\iota_{0}$ & $\odot$ & $\emptyset$ & 0 \\
\hline$\iota_{1}$ & $a_{\mathbf{t}_{0}}$ & $a_{\mathbf{t}_{0}} \mapsto\left(\{\}, \iota_{0}, 0\right)$ & 1 \\
\hline$\iota_{2}$ & $a_{\mathbf{t}_{0}}$ & $a_{\mathbf{t}_{0}} \mapsto\left(\{\}, \iota_{0}, 0\right)$ & 2 \\
\hline$\iota_{3}$ & $a_{\mathbf{t}_{0}}$ & $a_{\mathbf{t}_{0}} \mapsto\left(\{\mathbf{a}: 1\}, \iota_{0}, 0\right)$ & 3 \\
\hline$\iota_{4}$ & $a_{\mathbf{t}_{1}}$ & $\begin{array}{l}a_{\mathbf{t}_{0}} \mapsto\left(\{\mathbf{a}: 1\}, \iota_{0}, 0\right) \\
a_{\mathbf{t}_{1}} \mapsto\left(\{\}, \iota_{3}, 3\right)\end{array}$ & 4
\end{tabular}

and another trace executing the false branch is as follows:

\begin{tabular}{c|c|c|c}
$\sigma_{i}^{\mathbb{L}}$ & $\sigma_{i}^{\mathbb{E}}(\mathrm{obj})$ & $\sigma_{i}^{\mathbb{H}}$ & $\sigma_{i}^{\mathbb{D}}$ \\
\hline$\iota_{0}$ & $\odot$ & $\emptyset$ & 0 \\
\hline$\iota_{1}$ & $a_{\mathbf{f}_{0}}$ & $a_{\mathbf{f}_{0}} \mapsto\left(\{\}, \iota_{0}, 0\right)$ & 1 \\
\hline$l_{5}$ & $a_{\mathbf{f}_{0}}$ & $a_{\mathbf{f}_{0}} \mapsto\left(\{\}, \digamma_{0}, 0\right)$ & 2
\end{tabular}

\subsection{Concrete Semantics}

We define a small-step semantics characterized by a transition relation $\rightarrow$, and use the finite trace semantics induced by $\rightarrow$. The initial state is $\left(\mathscr{L}_{0},(\emptyset, \epsilon), \emptyset, 0\right)$ where $\mathscr{L}_{0}$ is the start control point of a given program. For instance, the transitions for simple variable creation and object allocation statements have the following semantics:

- Simple variable creation without initialization

$$
\begin{gathered}
\mathcal{l}_{0}: \quad \operatorname{var} \mathrm{x} ; \\
\mathcal{l}_{1}: \ldots \\
\left(\mathcal{C}_{0},\left(\sigma_{0}^{\mathbb{E}}, \sigma_{0}^{\mathbb{K}}\right), \sigma_{0}^{\mathbb{H}}, \sigma_{0}^{\mathbb{D}}\right) \rightarrow\left(\mathcal{C}_{1},\left(\sigma_{1}^{\mathbb{E}}, \sigma_{0}^{\mathbb{K}}\right), \sigma_{0}^{\mathbb{H}}, \sigma_{1}^{\mathbb{D}}\right) \text { where } \\
\sigma_{1}^{\mathbb{E}}=\sigma_{0}^{\mathbb{E}}[\mathrm{x} \mapsto \odot] \text { and } \sigma_{1}^{\mathbb{D}}=\sigma_{0}^{\mathbb{D}}+1
\end{gathered}
$$

- Object allocation

$$
\begin{array}{ll}
\complement_{0}: & \mathrm{x}=\{\} ; \\
\complement_{1}: & \ldots
\end{array}
$$

$\left(\mathcal{L}_{0},\left(\sigma_{0}^{\mathbb{E}}, \sigma_{0}^{\mathbb{K}}\right), \sigma_{0}^{\mathbb{H}}, \sigma_{0}^{\mathbb{D}}\right) \rightarrow\left(\mathcal{L}_{1},\left(\sigma_{1}^{\mathbb{E}}, \sigma_{0}^{\mathbb{K}}\right), \sigma_{1}^{\mathbb{H}}, \sigma_{1}^{\mathbb{D}}\right)$ where $a$ is a fresh address, $\sigma_{1}^{\mathbb{E}}=\sigma_{0}^{\mathbb{E}}[\mathrm{x} \mapsto a], \sigma_{1}^{\mathbb{H}}=\sigma_{0}^{\mathbb{H}}[a \mapsto$ $\left.\left(\{\}, \iota_{0}, \sigma_{0}^{\mathbb{D}}\right)\right]$, and $\sigma_{1}^{\mathbb{D}}=\sigma_{0}^{\mathbb{D}}+1$.

The remaining rules are available in a companion report [9]. Generally, the transition relation $\rightarrow$ should ensure that, for each transition $\left(\sigma_{0}^{\mathbb{L}}, \sigma_{0}^{\mathbb{C}}, \sigma_{0}^{\mathbb{H}}, \sigma_{0}^{\mathbb{D}}\right) \rightarrow\left(\sigma_{1}^{\mathbb{L}}, \sigma_{1}^{\mathbb{C}}, \sigma_{1}^{\mathbb{H}}, \sigma_{1}^{\mathbb{D}}\right)$, $\sigma_{1}^{\mathbb{D}}=\sigma_{0}^{\mathbb{D}}+1$.

\section{Recency Abstraction}

We formally define a series of abstractions towards recency abstraction on top of a given partition-based address abstraction. Then, we illustrate unintuitive behaviors of recency abstraction using two code examples.

\subsection{Abstractions}

We define a program abstraction by composing a series of abstractions:

- a classical flow-sensitive abstraction that maps each control state to the set of states that are observed at that location; and

- an abstraction of sets of states by collapsing addresses according to an address abstraction given as a parameter of the program abstraction.

Address abstraction An address abstraction is defined by:

- a set of abstract addresses $\mathbb{A}^{\sharp}$ (we note $a^{\sharp}$ for an element of this set); and

- a function $\phi^{\mathbb{A}}: \mathbb{A} \longrightarrow \mathbb{A}^{\sharp}$ that maps each address into the abstract address that represents it.

In the following, we consider several choices of this address abstraction. In each case, it fixes fully for each state the mapping between concrete addresses and abstract addresses.

State abstraction based on address abstraction Given a pair $\left(\mathbb{A}^{\sharp}, \phi^{\mathbb{A}}\right)$, we can define abstract domains and abstraction functions for the state abstraction as shown in Figure 2. Abstractions of control states and primitive values are their powersets. Because a power set of primitive values could be an infinite set, we should define its finite abstraction in real analysis, but we use powersets in this paper for the presentation brevity. Abstractions of states are a pair of abstractions of environments and heaps. An abstract environment is a map from variables to pairs of abstract addresses and sets of primitive values. An abstract heap is a map from abstract addresses to abstract objects. In the heap abstraction, we merge all the abstract objects corresponding to a given abstract address. Finally, an abstract object is a map from property names to pairs of abstract addresses and sets of primitive values or the special consider $\circledast$; when an abstract object $o^{\sharp}$ has a mapping from $\mathrm{p}$ to $\circledast$, it denotes that p may not exist in $o^{\sharp}$. Since abstract domains are complete lattices, we define at each step an element-wise abstraction function $\phi$, that maps each element to its best abstraction. Such functions implicitly define Galois connections. For instance, $\mathbb{A}, \phi^{\mathbb{A}}$ defines $\mathcal{P}(\mathbb{A}) \underset{\gamma}{\stackrel{\alpha}{\rightleftharpoons}} \mathbb{A}^{\sharp}$ by:

$\alpha\left(\mathbb{A}^{\prime}\right)=\bigsqcup_{a \in \mathbb{A}^{\prime}} \phi^{\mathbb{A}}(a), \quad \gamma\left(a^{\sharp}\right)=\left\{a \in \mathbb{A} \mid \phi^{\mathbb{A}}(a) \sqsubseteq a^{\sharp}\right\}$

Recency abstraction Recency abstraction is a commonly used address abstraction for JavaScript analysis, which is often defined on top of the allocation-site abstraction. We generalize the allocation-site abstraction as a partition-based address abstraction, and define recency abstraction on it. Thus, our formalization can represent recency abstraction 


\begin{tabular}{|c|c|c|}
\hline Concrete domain & Abstract domain & Element-wise abstraction function \\
\hline $\mathbb{S}$ & $\mathbb{S}^{\sharp}=\mathbb{E}^{\sharp} \times \mathbb{H}^{\sharp}$ & $\phi^{\mathbb{S}}\left(\left(-,(e,-), h,_{-}\right)\right)=\left(\phi^{\mathbb{E}}(e), \phi^{\mathbb{H}}(h)\right)$ \\
\hline $\mathbb{E}$ & $\mathbb{E}^{\sharp}=\mathbb{X} \longrightarrow \mathbb{A}^{\sharp} \times \mathcal{P}\left(\mathbb{V}_{\mathrm{p}}\right)$ & $\phi^{\mathbb{E}}(e)=\lambda(\mathrm{x} \in \operatorname{Domain}(e)) \cdot \begin{cases}\left(\phi^{\mathbb{A}}(e(\mathrm{x})),\{\}\right) & \text { if } e(\mathrm{x}) \text { is an address } \\
(\perp,\{e(\mathrm{x})\}) & \text { if } e(\mathrm{x}) \text { is a primitive value }\end{cases}$ \\
\hline $\mathbb{H}$ & $\mathbb{H}^{\sharp}=\mathbb{A}^{\sharp} \longrightarrow \mathbb{O}^{\sharp}$ & $\phi^{\mathbb{H}}(h)=\lambda\left(a^{\sharp} \in \mathbb{A}^{\sharp}\right) \cdot \sqcup\left\{\phi^{\oplus}(o) \mid \exists a \in \mathbb{A}, \phi^{\mathbb{A}}(a)=a^{\sharp} \wedge h(a)=\left(o,,_{-},\right)\right\}$ \\
\hline (1) & $\mathbb{O}^{\sharp}=\mathbb{P} \longrightarrow \mathbb{A}^{\sharp} \times \mathcal{P}\left(\mathbb{V}_{\mathrm{p}} \uplus\{\circledast\}\right)$ & $\phi^{\mathbb{O}}(o)=\lambda(\mathrm{p}) \cdot \begin{cases}\left(\phi^{\mathbb{A}}(o(\mathrm{p})),\{\}\right) & \text { if } o(\mathrm{p}) \text { is an address } \\
(\perp,\{o(\mathrm{p})\}) & \text { if } o(\mathrm{p}) \text { is a primitive value } \\
(\perp,\{\circledast\}) & \text { if } \mathrm{p} \notin \text { Domain }(o)\end{cases}$ \\
\hline
\end{tabular}

Figure 2. Abstractions based on the address abstraction $\left(\mathbb{A}^{\sharp}, \phi^{\mathbb{A}}\right)$

on heap cloning [6], which is yet another partition-based address abstraction.

A partition-based address abstraction $\left(\mathbb{A}_{\delta}^{\sharp}, \phi_{\delta}^{\mathbb{A}}\right)$ is defined with a partition $\delta: \mathbb{A} \rightarrow \Pi$ where $\mathbb{A}_{\delta}^{\sharp}=\mathcal{P}(\Pi)$ and $\phi_{\delta}^{\mathbb{A}}(a)=\{\delta(a)\}$. We could simplify the heap abstraction using the partition $\delta$ as follows:

- $\mathbb{H}_{\delta}^{\sharp}=\Pi \longrightarrow \mathbb{O}^{\sharp}$

- $\phi_{\delta}^{\mathbb{H}}(h)=\lambda(\pi \in \Pi) \cdot \bigsqcup\left\{\phi^{\mathbb{O}}(o) \mid \exists a \in \mathbb{A}, \delta(a)=\pi \wedge\right.$ $\left.h(a)=\left(o,,_{-},\right)\right\}$

Given a partition-based address abstraction with a partition $\delta: \mathbb{A} \rightarrow \Pi$, and a corresponding state $(\mathcal{L}, c, h, n)$, we define recency abstraction as follows:

- $\mathbb{A}_{\mathrm{r}[\delta]}^{\sharp}=\mathcal{P}(\Pi \times\{\mathbf{r}, \mathbf{o}\})$;

- $\phi_{\mathrm{r}[\delta]}^{\mathbb{A}}(a)= \begin{cases}\{(\pi, \mathbf{r})\} & \text { if } h(a)=\left(o, \mathcal{L}, n_{r}\right) \\ \{(\pi, \mathbf{o})\} & \text { otherwise }\end{cases}$

where $\pi=\delta(a)$

and $n_{r}=\max \left\{n^{\prime} \mid \exists a^{\prime} \in \mathbb{A}, o^{\prime} \in \mathbb{O}, l^{\prime} \in \mathbb{L}\right.$, $\left.\delta\left(a^{\prime}\right)=\pi \wedge h\left(a^{\prime}\right)=\left(o^{\prime}, l^{\prime}, n^{\prime}\right)\right\}$.

This allows to abstract sets of states similarly as above except that the address abstraction function depends on states.

\subsection{Unintuitive Behaviors of Recency Abstraction}

Now, we illustrate unintuitive behaviors of recency abstraction using two examples.

Example 1 The code in Figure 1 contains two allocationsites $\zeta_{0}$ and $\mathcal{l}_{3}$. Let us consider two partition-based address abstractions: the allocation-site abstraction with $\delta_{i d}: \mathbb{A} \rightarrow$ $\mathbb{L}$, which divides addresses based on their allocation sites and a crude one with $\delta_{\top}: \mathbb{A} \rightarrow\{\pi\}$ for some $\pi$, which does not partition at all. Clearly, the abstraction $\left(\mathbb{A}_{\delta_{i d}}^{\sharp}, \phi_{\delta_{i d}}^{\mathbb{A}}\right)$ defines a more precise address partition than $\left(\mathbb{A}_{\delta_{\tau}}^{\sharp}, \phi_{\delta_{\tau}}^{\mathbb{A}}\right)$. Unfortunately, recency abstraction does not preserve this "more precise than" relationship. Let's look at the analysis results at the control state $l_{4}$. The abstraction with recency abstraction $\left(\mathbb{A}_{\mathrm{r}\left[\delta_{\mathrm{id}}\right]}^{\sharp}, \phi_{\mathrm{r}\left[\delta_{\mathrm{id}}\right]}^{\mathbb{A}}\right)$ produces the following result:

\begin{tabular}{c|c|l} 
& \multicolumn{1}{|c|}{$e^{\sharp}$} & \multicolumn{1}{c}{$h^{\sharp}$} \\
\hline true branch & obj $\mapsto\left\{\left(\mathscr{C}_{3}, \mathbf{r}\right)\right\}$ & $\begin{array}{l}\left(\mathscr{L}_{0}, \mathbf{r}\right) \mapsto\{\mathrm{a} \mapsto\{1\}\} \\
\left(\mathscr{L}_{3}, \mathbf{r}\right) \mapsto\{\}\end{array}$ \\
\hline false branch & obj $\mapsto\left\{\left(\mathscr{L}_{0}, \mathbf{r}\right)\right\}$ & $\left(\mathscr{L}_{0}, \mathbf{r}\right) \mapsto\{\}$ \\
\hline join & obj $\mapsto\left\{\left(\mathscr{L}_{0}, \mathbf{r}\right)\right.$, & $\left(\mathscr{L}_{0}, \mathbf{r}\right) \mapsto\{\mathrm{a} \mapsto\{\circledast, 1\}\}$ \\
& $\left.\left(\mathscr{C}_{3}, \mathbf{r}\right)\right\}$ & $\left(\mathscr{C}_{3}, \mathbf{r}\right) \mapsto\{\}$
\end{tabular}

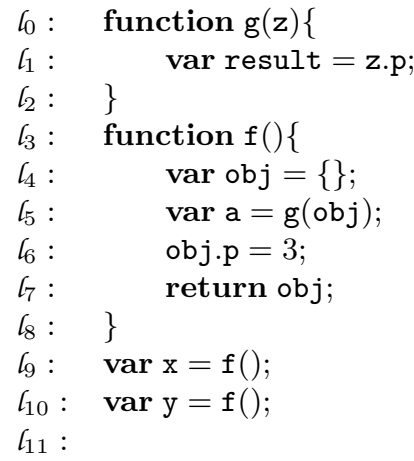

Figure 3. Recency abstraction interfering with sensitivities

The joined result of both true and false branches shows that obj.a may have values $\{\circledast, 1\}$. On the contrary, the abstraction with $\left(\mathbb{A}_{\mathrm{r}\left[\delta_{T}\right]}^{\sharp}, \phi_{\mathrm{r}\left[\delta_{T}\right]}^{\mathbb{A}}\right)$ produces the following:

\begin{tabular}{c|c|c} 
& $e^{\sharp}$ & \multicolumn{1}{c}{$h^{\sharp}$} \\
\hline true branch & obj $\mapsto\{(\pi, \mathbf{r})\}$ & $\begin{array}{l}(\pi, \mathbf{r}) \mapsto\{\} \\
(\pi, \mathbf{o}) \mapsto\{\mathrm{a} \mapsto\{1\}\}\end{array}$ \\
\hline false branch & $\mathrm{obj} \mapsto\{(\pi, \mathbf{r})\}$ & $(\pi, \mathbf{r}) \mapsto\{\}$ \\
\hline join & $\mathrm{obj} \mapsto\{(\pi, \mathbf{r})\}$ & $\begin{array}{l}(\pi, \mathbf{r}) \mapsto\{\} \\
(\pi, \mathbf{o}) \mapsto\{\mathrm{a} \mapsto\{1\}\}\end{array}$
\end{tabular}

The joined result of both branches shows that a does not exist in obj; thus, the value of obj.a is $\{\circledast\}$. This example shows that $\left(\mathbb{A}_{\mathrm{r}\left[\delta_{T}\right]}^{\sharp}, \phi_{\mathrm{r}\left[\delta_{T}\right]}^{\mathbb{A}}\right)$ is more precise than $\left(\mathbb{A}_{\mathrm{r}\left[\delta_{\mathrm{id}}\right]}^{\sharp}, \phi_{\mathrm{r}\left[\delta_{\mathrm{id}}\right]}^{\mathbb{A}}\right)$ while $\left(\mathbb{A}_{\delta_{i d}}^{\sharp}, \phi_{\delta_{i d}}^{\mathbb{A}}\right)$ is more precise than $\left(\mathbb{A}_{\delta_{T}}^{\sharp}, \phi_{\delta_{T}}^{\mathbb{A}}\right)$. Therefore, the precision relationship of the underlying address abstraction is not preserved with recency abstraction.

Example 2 The code in Figure 3 shows that recency abstraction may interfere with analysis sensitivities. Let us consider two analysis sensitivities: 1-CFA that distinguishes the same function from its different call sites using its caller, and 0-CFA that does not distinguish different call sites of the same function. Then, we consider the allocation-site abstraction refined by different sensitivities. With 1-CFA, the partition is $\delta: \mathbb{A} \rightarrow\left\{l_{4 / 9}, l_{4 / 10}\right\}$ where $\ell_{4 / 9}$ means that the allocation-site $\ell_{4}$ with the call-site $l_{9}$ and $\ell_{4 / 10}$ means that the allocation-site $l_{4}$ with the call-site $\mathscr{l}_{10}$. In this case, we get the 
following result at the control state $C_{1}$ :

\begin{tabular}{|c|c|c|}
\hline & $e^{\sharp}$ & $h^{\sharp}$ \\
\hline call $l_{9}, l_{5}$ & $\mathbf{z} \mapsto\left\{\left(l_{4 / 9}, \mathbf{r}\right)\right\}$ & $\left(\mathcal{l}_{4 / 9}, \mathbf{r}\right) \mapsto\{\}$ \\
\hline call $\mathcal{l}_{10}, \mathcal{L}_{5}$ & $\mathbf{z} \mapsto\left\{\left(\mathcal{l}_{4 / 10}, \mathbf{r}\right)\right\}$ & $\begin{array}{l}\left(\mathcal{L}_{4 / 9}, \mathbf{r}\right) \mapsto\{\mathrm{p} \mapsto\{3\}\} \\
\left(\mathcal{L}_{4 / 10}, \mathbf{r}\right) \mapsto\{\}\end{array}$ \\
\hline join & $\begin{aligned} \mathbf{z} \mapsto\{ & \left(l_{4 / 9}, \mathbf{r}\right), \\
& \left.\left(l_{4 / 10}, \mathbf{r}\right)\right\}\end{aligned}$ & $\begin{array}{l}\left(\mathcal{l}_{4 / 9}, \mathbf{r}\right) \mapsto\{\mathrm{p} \mapsto\{\circledast, 3\}\} \\
\left(\mathcal{l}_{4 / 10}, \mathbf{r}\right) \mapsto\{\}\end{array}$ \\
\hline
\end{tabular}

With 0-CFA, the partition is $\delta: \mathbb{A} \rightarrow\left\{c_{4}\right\}$. Thus, it has only one partition and we get the following result at the control state $\zeta_{1}$ :

\begin{tabular}{|c|c|c|}
\hline & $e^{\sharp}$ & $h^{\sharp}$ \\
\hline call $l_{9}, l_{5}$ & $\mathbf{z} \mapsto\left\{\left(\mathcal{l}_{4}, \mathbf{r}\right)\right\}$ & $\left(\mathcal{l}_{4}, \mathbf{r}\right) \mapsto\{\}$ \\
\hline call $C_{10}, \mathcal{L}_{5}$ & $\mathbf{z} \mapsto\left\{\left(\mathcal{l}_{4}, \mathbf{r}\right)\right\}$ & $\begin{array}{l}\left(\mathcal{l}_{4}, \mathbf{r}\right) \mapsto\{\} \\
\left(\mathcal{L}_{4}, \mathbf{o}\right) \mapsto\{\mathrm{p} \mapsto\{3\}\}\end{array}$ \\
\hline join & $\mathbf{z} \mapsto\left\{\left(\mathcal{l}_{4}, \mathbf{r}\right)\right\}$ & $\begin{array}{l}\left(\mathcal{l}_{4}, \mathbf{r}\right) \mapsto\{\} \\
\left(\mathcal{L}_{4}, \mathbf{o}\right) \mapsto\{\mathrm{p} \mapsto\{3\}\}\end{array}$ \\
\hline
\end{tabular}

This example shows that a more precise 1-CFA may produce less precise results than 0-CFA when combined with recency abstraction. Therefore, the precision relationship of analysis sensitivities is not preserved when combined with recency abstraction.

\section{Singleton Abstraction}

In this section, we explain the unintuitive behaviors of recency abstraction in terms of the refinement relationship between partition-based address abstractions. Then, we present singleton abstraction, a new heap abstraction based on a given partition-based address abstraction, which preserves the refinement relationship of its underlying address abstraction and moreover allows strong updates on singleton addresses.

\subsection{Refinement of Address Abstraction}

We first define terminologies to discuss the behaviors of recency abstraction. A partition-based address abstractions $\left(\mathbb{A}_{\delta_{i}}^{\sharp}, \phi_{\delta_{i}}^{\mathbb{A}}\right)$ is defined with a partition $\delta_{i}: \mathbb{A} \rightarrow \Pi_{i}$. A partition-based address abstraction is a refinement of another, if and only if their partitions have the refinement relationship accordingly.

Definition $1(\preceq)$. $\left(\mathbb{A}_{\delta_{1}}^{\#}, \phi_{\delta_{1}}^{\mathbb{A}}\right) \preceq\left(\mathbb{A}_{\delta_{2}}^{\#}, \phi_{\delta_{2}}^{\mathbb{A}}\right)$ iff $\delta_{1}$ is a refinement partition of $\delta_{2}$.

An address abstraction $\left(\mathbb{A}_{1}^{\#}, \phi_{1}^{\mathbb{A}}\right)$ is more precise than $\left(\mathbb{A}_{2}^{\sharp}, \phi_{2}^{\mathbb{A}}\right)$ if and only if the concretization of the former is a subset of that of the latter.

Definition $2\left(\preceq_{p}\right)$. $\left(\mathbb{A}_{1}^{\sharp}, \phi_{1}^{\mathbb{A}}\right) \preceq_{p}\left(\mathbb{A}_{2}^{\sharp}, \phi_{2}^{\mathbb{A}}\right)$ iff $\gamma_{1} \circ \alpha_{1} \subseteq$ $\gamma_{2} \circ \alpha_{2}$.

Then, we prove that the refinement relation implies the precision relation.

Theorem 1 (Implication of precision from refinement).

$$
\left(\mathbb{A}_{\delta_{1}}^{\#}, \phi_{\delta_{1}}^{\mathbb{A}}\right) \preceq\left(\mathbb{A}_{\delta_{2}}^{\#}, \phi_{\delta_{2}}^{\mathbb{A}}\right) \Rightarrow\left(\mathbb{A}_{\delta_{1}}^{\#}, \phi_{\delta_{1}}^{\mathbb{A}}\right) \preceq_{p}\left(\mathbb{A}_{\delta_{2}}^{\#}, \phi_{\delta_{2}}^{\mathbb{A}}\right)
$$

Now, let us revisit the first example in Section 3.2 with the refinement relation. Because $\delta_{i d}$ is a partition of $\delta_{\top}$, we have $\left(\mathbb{A}_{\delta_{i d}}^{\#}, \phi_{\delta_{i d}}^{\mathbb{A}}\right) \preceq\left(\mathbb{A}_{\delta_{\top}}^{\#}, \phi_{\delta_{\top}}^{\mathbb{A}}\right)$. The recency abstraction with a cruder partition $\left(\mathbb{A}_{\mathrm{r}\left[\delta_{\top}\right]}^{\sharp}, \phi_{\mathrm{r}\left[\delta_{\top}\right]}^{\mathbb{A}}\right)$ has two partitions $(\pi, \mathbf{r})$ and $(\pi, \mathbf{o}): \gamma((\pi, \mathbf{r}))=\left\{a_{\mathbf{t}_{1}}, a_{\mathbf{f}_{0}}\right\}$ and $\gamma((\pi, \mathbf{o}))=\left\{a_{\mathbf{t}_{0}}\right\}$ where $a_{\mathbf{t}_{0}}$ and $a_{\mathbf{t}_{1}}$ are concrete addresses created at $\varepsilon_{0}$ and $l_{3}$, respectively, for the true branch, and $a_{\mathbf{f}_{0}}$ is a concrete address created at $\ell_{0}$ for the false branch. The other recency abstraction $\left(\mathbb{A}_{\mathrm{r}\left[\delta_{\mathrm{id}}\right]}^{\#}, \phi_{\mathrm{r}\left[\delta_{\mathrm{id}}\right]}^{\mathbb{A}}\right)$ has four partitions, and only two partitions $\left(\mathcal{L}_{0}, \mathbf{r}\right)$ and $\left(\mathcal{L}_{3}, \mathbf{r}\right)$ have elements: $\gamma\left(\left(\mathcal{L}_{0}, \mathbf{r}\right)\right)=\left\{a_{\mathbf{t}_{0}}, a_{\mathbf{f}_{0}}\right\}$ and $\gamma\left(\left(\mathcal{L}_{3}, \mathbf{r}\right)\right)=\left\{a_{\mathbf{t}_{1}}\right\}$. Thus, $\left(\mathbb{A}_{\mathrm{r}\left[\delta_{\mathrm{id}}\right]}^{\sharp}, \phi_{\mathrm{r}\left[\delta_{\mathrm{id}}\right]}^{\mathbb{A}}\right) \npreceq\left(\mathbb{A}_{\mathrm{r}\left[\delta_{\top}\right]}^{\sharp}, \phi_{\mathrm{r}\left[\delta_{\top}\right]}^{\mathbb{A}}\right)$, which illustrates a case where recency abstraction does not preserve the refinement relation of its underlying address abstraction, which in turn does not preserve their precision relation. Similarly, the second example shows that recency abstraction with a more precise 1-CFA analysis sensitivity does not always produce more precise analysis results than recency abstraction with a less precise 0-CFA.

\subsection{Singleton Abstraction}

To alleviate the problem, we decide not to divide a given partition but to simply perform strong updates on singleton objects. Thus, we propose singleton abstraction, a new heap abstraction that preserves the refinement relationship of its underlying address abstraction. It can provide more precise analysis results with more precise underlying address abstractions and with more precise analysis sensitivities.

Given a partition-based address abstraction $\left(\mathbb{A}_{\delta}^{\sharp}, \phi_{\delta}^{\mathbb{A}}\right)$ with a partition $\delta=\mathbb{A} \rightarrow \Pi$, we define singleton abstraction as follows:

- $\mathbb{H}_{\mathrm{s}[\delta]}^{\sharp}=\Pi \longrightarrow \mathbb{O}^{\sharp} \times\{\mathbf{s}, \mathbf{m}\}$;

- $\phi_{\mathrm{s}[\delta]}^{\mathbb{H}}(h)=\lambda(\pi \in \Pi) \cdot\left(\phi_{\delta}^{\mathbb{H}}(\pi),\left\{\begin{array}{ll}\mathbf{s} & \text { if }|U|=1 \\ \mathbf{m} & \text { otherwise }\end{array}\right)\right.$

where $U=\left\{a^{\prime} \in \mathbb{A} \mid \delta\left(a^{\prime}\right)=\pi \wedge a^{\prime} \in \operatorname{Domain}(h)\right\}$.

It distinguishes partitions with only one address as $\mathbf{s}$ and maps the other partitions to $\mathbf{m}$. Merging two mappings from the same partition to both singleton (s) and multiple (m) results in $\mathbf{m}$.

Unlike recency abstraction, the singleton abstraction preserves the refinement relation of its underlying address abstraction because they use the same partition from the underlying address abstraction. While the expressive power of the singleton abstraction is the same as its partition-based address abstraction, singleton abstraction allows strong updates for address partitions that map to s. It permits strong updates on objects created at specific allocation sites.

\section{Evaluation}

We evaluate the precision of singleton abstraction in comparison with recency abstraction. We conducted experiments with 3 sets of benchmarks-JSAI, SunSpider, and V8-consisting of 24 programs on a $2.8 \mathrm{GHz}$ Intel Core i5 iMac with $16 \mathrm{~GB}$ memory. We implemented 3 address 


\begin{tabular}{|c|c|c|c|c|c|}
\hline Bench & Program & LOC & Recency & Singleton & Total \\
\hline \multirow{7}{*}{ JSAI } & adn-chess.js & 234 & 90 & 55 & 127 \\
\hline & adn-coffee_pods_deals.js & 367 & 45 & 37 & 141 \\
\hline & adn-less_spam_please.js & 759 & 213 & 143 & 432 \\
\hline & adn-live_pagerank.js & 882 & 132 & 117 & 323 \\
\hline & adn-odesk_job_watcher.js & 168 & 56 & 52 & 71 \\
\hline & adn-pinpoints.js & 548 & 58 & 57 & 232 \\
\hline & adn-tryagain.js & 929 & 103 & 72 & 525 \\
\hline \multirow{14}{*}{ SunSpider } & 3d-morph.js & 23 & 1 & 1 & $\overline{4}$ \\
\hline & access-binary-trees.js & 38 & 14 & 10 & 16 \\
\hline & access-fannkuch.js & 51 & 1 & 1 & 19 \\
\hline & access-nbody.js & 142 & 32 & 15 & 78 \\
\hline & access-nsieve.js & 28 & 2 & 0 & 4 \\
\hline & bitops-3bit-bits-in-byte.js & 13 & 0 & 0 & 0 \\
\hline & bitops-bits-in-byte.js & 14 & 0 & 0 & 0 \\
\hline & bitops-bitwise-and.js & 3 & 0 & 0 & 0 \\
\hline & bitops-nsieve-bits.js & 22 & 1 & 1 & 7 \\
\hline & controlflow-recursive.js & 18 & 0 & 0 & $\overline{0}$ \\
\hline & math-cordic.js & 53 & 4 & 4 & 6 \\
\hline & math-partial-sums.js & 25 & 4 & 4 & 4 \\
\hline & math-spectral-norm.js & 41 & 2 & 1 & 16 \\
\hline & string-fasta.js & 70 & 15 & 10 & 18 \\
\hline \multirow{3}{*}{ V8 } & navier-stokes.js & 331 & 36 & 17 & 92 \\
\hline & richards.js & 288 & 119 & 117 & 197 \\
\hline & splay.js & 205 & 108 & 108 & 132 \\
\hline \multicolumn{3}{|c|}{ Total } & 1036 & 831 & 2,444 \\
\hline \multicolumn{3}{|c|}{ Ratio (\%) } & 42.39 & 33.63 & - \\
\hline
\end{tabular}

Table 1. Analysis precision

abstractions-allocation-site abstraction, recency abstraction, and singleton abstraction-on an open-source JavaScript static analysis framework, SAFE [7]. The implemented recency abstraction and singleton abstraction are built on top of the allocation-site abstraction.

The analyses took on average 86.92, 122.73, and 79.77 seconds for the allocation-site, recency, and singleton abstractions, respectively. It means that singleton abstraction does not incur much performance overhead like recency abstraction while providing comparable analysis precision with recency abstraction. We observed that the more complex benchmark programs get, the more performance overhead recency abstraction causes.

For the analysis precision, we compare the numbers of object property loads like obj.p that have more precise results with recency or singleton abstraction compared with just the allocation-site abstraction. Table 1 summarizes the experimental results; the 3 rd column shows the lines of code, the 4th and the 5th columns show the numbers of more precise property loads by recency and singleton abstractions, respectively, and the last column shows the total number of property loads in each program. For example, the first program in the JSAI benchmarks, adn-chess.js, has 127 property loads, among which recency abstraction analzyes 90 property loads more precisely than the allocation-site abstraction. In summary, recency and singleton abstractions analyze about $42.39 \%$ and $33.63 \%$ of property loads more precisely on average, respectively. Note that recency abstraction divides partitions into two parts: recent and old. Therefore, recency abstraction provides more precise analysis re- sults than singleton abstraction when programs update recent addresses and their allocation sites also have old addresses pointing to different shapes of objects. We plan to extend the set of benchmark programs to understand the relationships between recency and singleton abstractions more clearly.

\section{Conclusion}

We revisited recency abstraction, a typical address abstraction technique for static analysis of JavaScript programs. We formally defined it on a partition-based address abstraction, and we used the formalization to describe unintuitive behaviors of recency abstraction. We explained the behaviors by showing that recency abstraction does not preserve the refinement relationship between its underlying address abstractions. Thus, it is difficult to predict which address abstraction would provide the most precise analysis result for recency abstraction. Thus, we proposed singleton abstraction, a new heap abstraction using a partition-based abstraction. It preserves the refinement relationship of the underlying address abstractions. Therefore, it is compositional with other analysis techniques. Moreover, our experiments showed that it provides similar analysis precision with recency abstraction while reducing the performance overhead.

\section{Acknowledgment}

This research has received funding from the European Research Council under the EU FP 7, grant Agreement 278673, Project MemCAD, and National Research Foundation of Korea (Grant NRF-2014R1A2A2A01003235).

\section{References}

[1] TIOBE Index for February 2017. http://www.tiobe.com/ tiobe-index.

[2] Iot.js: A framework for Internet of Things. http://samsung.github.io/jerryscript/, 2015.

[3] E. Andreasen and A. Møller. Determinacy in static analysis for jQuery. In OOPSLA, 2014.

[4] G. Balakrishnan and T. Reps. Recency-abstraction for heap-allocated storage. In SAS, 2006.

[5] S. H. Jensen, A. Møller, and P. Thiemann. Type analysis for JavaScript. In SAS, 2009.

[6] C. Lattner, A. Lenharth, and V. Adve. Making context-sensitive points-to analysis with heap cloning practical for the real world. In PLDI, 2007.

[7] H. Lee, S. Won, J. Jin, J. Cho, and S. Ryu. SAFE: Formal specification and implementation of a scalable analysis framework for ECMAScript. In FOOL, 2012.

[8] C. Park and S. Ryu. Scalable and precise static analysis of JavaScript applications via loop-sensitivity. In ECOOP, 2015.

[9] J. Park, X. Rival, and S. Ryu. Revisiting recency abstraction for JavaScript (extended). http://plrg.kaist.ac.kr/doku.php?id=research:material, 2017.

[10] M. Schäfer, M. Sridharan, J. Dolby, and F. Tip. Dynamic determinacy analysis. In PLDI, 2013.

[11] M. Sridharan, J. Dolby, S. Chandra, M. Schäfer, and F. Tip. Correlation tracking for points-to analysis of JavaScript. In ECOOP, 2012. 